\title{
Neoadjuvant Treatment for Resectable, Stage IIIA Non-Small Cell Lung Cancer
}

\author{
Ivane Kiladze ${ }^{1,4 *}$, Merab Kiladze ${ }^{2,4}$ and Branislav Jeremic ${ }^{3}$ \\ ${ }^{1}$ Department of Clinical Oncology, Caucasus Medical Centre, Georgia, \\ ${ }^{2}$ Department of Surgery, Clinic “Gidmedi", Georgia \\ ${ }^{3}$ Radiation Oncology Department, Research Institute of Clinical Medicine after academician F.Todua, Georgia \\ ${ }^{4}$ Ivane Javakhishvili Tbilisi State University, Tbilisi, Georgia
}

Submission: January 17, 2021; Published: January 25, 2021

*Corresponding author: Ivane Kiladze, MD. Department of Clinical Oncology, Caucasus Medical Centre 23, P. Kavtaradze Str. 0186. Tbilisi, Georgia

\begin{abstract}
The optimal treatment of patients with stage IIIA non-small cell lung cancer (NSCLC) remains highly controversial. In resectable stage IIIA NSCLC, there is still a considerable debate regarding the best strategy. Treatment choice is often a function of institutional and/or physician preference. Treatment consists of neo-adjuvant chemotherapy or chemoradiotherapy (CHRT) followed by surgery with or without postoperative radiotherapy (RT), definitive CHRT, or neoadjuvant immunotherapy followed by surgery and several other options. Neo-adjuvant therapy for operable NSCLC has been the subject of a large number of studies in the literature. We summarized existing data and also highlight ongoing trials, focusing on neoadjuvant immunotherapy. Neoadjuvant CHRT seems to be safe and efficient and is associated with an improved pathological outcome, but it has failed to demonstrate any advantage in terms of progression-free survival or overall survival compared to neoadjuvant chemotherapy. Contrary to previous fears, radiotherapy does not add a higher toxicity, nor does it increase post-operative mortality compared to chemotherapy alone. Studies with chemoimmunotherapy provide a higher rate of pathologic responses and downstaging compared to chemotherapy. On the other hand, it remains to be confirmed whether pCR is a sufficient surrogate for OS.
\end{abstract}

Results of ongoing neoadjuvant immunotherapy trials are awaiting and we believe, the next decades will bring much needed improvements for patients. Still the controversy is not being solved and further trials considering a better patients' selection, innovative radiotherapy and more efficient systemic treatments need to be undertaken.

Keywords: NSCLC; Stage IIIA; Neoadjuvant treatment; Chemotherapy; Chemoradiotherapy; Surgery; Immunotherapy

Abbreviations: NSCLC: Non-small Cell Lung Cancer; CHRT- Chemoradiation; CHT: Chemotherapy; RT: Radiotherapy; pCR: Pathological Complete Response; pRR: Pathological Response Rates; RND: Residual Nodal Disease; N-PCR: Nodal Pathologic Complete Response; TMT: Trimodality Treatment; DMFS: Distant Metastasis-Free Survival; MST: The Median Survival Time; CR: Complete Resection; MPR: Major pathologic response

\section{Introduction}

The treatment of stage IIIA non-small cell lung cancer (NSCLC) remains one of the major areas of controversy in thoracic oncology. Patients with stage IIIA disease represent a heterogeneous group due to various extents of their tumor (T), and lymph node $(\mathrm{N})$ status as well as frequent co-morbidities, hence, different classification approaches in this setting have been adopted. One pragmatic approach is to classify stage IIIA disease by resectability and operability. The current standard of care for unresectable/inoperable stage IIIA patients is definitive chemoradiation (CHRT) with a platinum doublet which is nowadays frequently followed by consolidation durvalumab [1].

In resectable stage IIIA NSCLC, however, there is still a considerable debate regarding the best strategy, including surgery with either adjuvant or neoadjuvant chemo- and/or radiotherapy (RT) or definitive concurrent CHRT and several other strategies. Neo-adjuvant therapy for operable NSCLC has been the subject of a large number of studies in the literature, and in spite of progress in this field, many issues remain unsolved. 


\section{Cancer Therapy \& Oncology International Journal}

Neoadjuvant therapy has several theoretical advantages as in vivo assessment of response to chemotherapy (CHT) or CHRT helping to identify patients who will potentially benefit due to, early treatment of micrometastatic disease, potential downstaging with increasing resectability and the possibility of identification of surrogate clinical and biological markers that may correlate with response to therapy and potential long-term outcome. Also, neoadjuvant treatments are aimed at improving the overall outcome decreasing the rate of local failures and distant metastases observed after surgery alone [2]. The present manuscript reviews past studies and it also highlights ongoing trials including neoadjuvant immunotherapy.

\section{Discussion}

\section{Neoadjuvant Chemotherapy Followed by Surgery}

The role for neoadjuvant CHT before surgery remains controversial. Several trials [3-5] have shown improved survival but all of them were with small patient numbers. However, the study of Gilligan and colleagues showed only a trend favoring neoadjuvant CHT in the subset of patients with resectable stage III NSCLC, but without statistical significance on survival [6]. Metaanalysis Collaborative Group on neo-adjuvant CHT for NSCLC has collected individual participant data from 2,385 patients included in 15 controlled randomized trials. Most patients included were stage IB-IIIA. The results showed a $13 \%$ reduction in the relative risk of death, with an absolute survival improvement of $5 \%$ at 5 years, from $40 \%$ to $45 \%$. Looking at the first events, local recurrence occurred in $24 \%$, distant recurrence in $31 \%$ and both local and distant recurrence in $9 \%$. In this meta-analysis, stage did not seem to alter the effect of CHT [7]. In addition, positive effect of CHT was also observed in another meta-analysis, looking specifically at 8 studies on stage III, where the improvement in OS with CHT was also statistically significant [8].

While systematic reviews and meta-analysis from randomized controlled trials have suggested that it improves survival [9-12], disadvantages of these investigations are different combinations of trials (patients, treatments), making the specific effects of preoperative CHT difficult to discern. However, in many trials, the pathological complete response (pCR) rate was low, and the local-regional recurrence rate was high. In three more recent randomized trials comparing neoadjuvant $\mathrm{CHT}$ followed by surgery to surgery alone in stage III NSCLC, the complete pCR in the induction arm was only between $6 \%$ and $10.5 \%[13,14]$. As pCR is an indicator of response and a possible surrogate for survival, it seems logical to improve pCR by an additional locoregional treatment to surgery such as RT. For these different reasons, it appears that the addition of RT to CHT in neo-adjuvant strategies deserves additional consideration.

\section{Neoadjuvant CHRT Followed by Surgery}

A large number of retrospective studies on neo-adjuvant CHRT in patients with operable stage IIIA NSCLC have been published, using mostly cisplatin doublets while RT schedules mainly included conventional fractionation schemes with a few hyperfractionated schemes, with doses between 43-60 Gy [15-22]. The pCR, when reported, varied 16\%-27 \% [15,17-20,22], except in one study where it was as high as $40 \%$ [21]. The median survival time (MST) ranged between 21 and 36 months, and the 5-year overall survival (OS) ranged 31\%-40\% [15-22]. Some studies showed association of higher pCR with an increased survival $[15,16,21,22]$, however results from these retrospective studies are to be interpreted with caution due to patients' selection and other existing biases.

The study by the German Lung Cancer Cooperative Group assessed the additional effect of preoperative CHRT on tumour resection, pathological response rates (pRR), and survival in patients with stage IIIA-IIIB NSCLC [23]. In both groups' patients received three cycles of cisplatin and etoposide, followed by CHRT and then surgical resection (intervention group) or surgery, and then further RT (control group). Study showed no significant difference in either PFS or OS between treatment groups. However, preoperative CHRT in patients undergoing surgery significantly increased the proportion of those with complete resection (CR); and in those with $\mathrm{CR}$, preoperative $\mathrm{CHT}$ increased the proportion of patients with mediastinal downstaging and histopathological response. Significant increase in oesophagitis and haematological toxicity was observed in the interventional group, whereas pneumonitis increased significantly in the control group and contributed substantially to RT-related mortality in that group. A caveat against pneumonectomy was stated since treatment-related mortality reached $14 \%$ in patients treated by pneumonectomy [23].

In another landmark trial, Albain et al. compared concurrent induction CHRT (cisplatin-etoposide, 45 Gy) followed by surgery versus definitive concurrent CHRT(61 Gy) in potentially resectable patients [24], This trial (INT0139), showed that surgical resection following induction CHRT results in improved local control and PFS in appropriately selected patients with stage IIIA NSCLC. However, this did not translate into an OS benefit, possibly because of treatment-related morbidity in the surgical arm. On subset, post-hoc analysis, when comparing those who underwent a lobectomy only to definitive CHRT patients, there was an improvement in MST of 33.6 months vs. 21.7 months and 5 -year OS of $36 \%$ vs. $18 \%$ ( $p=0.002$ ) in the lobectomy and CHRT arms, respectively. The percentage of death after pneumonectomy was $7,9 \%$, which, however, rose to $26 \%$ when neoadjuvant CHRT was followed by right pneumonectomy. Thus, appropriate (i.e. limited) surgical resection after induction treatment seems to be an option in a selection of stage IIIA NSCLC patients, lobectomy with systemic mediastinal lymph nodes dissection being preferred over pneumonectomy because the latter carries higher surgical risk after CHRT [24].

Some Japanese studies $[25,26]$ have reported a high rate of downstaging in stage IIIA patients treated with induction CHRT including S-1 and cisplatin-followed by surgery. These series 
also included pneumonectomies without increased mortality. Although the reported rates of 5-year OS are good (around 60\%), it is likely that these results were positively biased by very strict case selection criteria. The favorable outcomes for patients undergoing neoadjuvant CHRT followed by non-pneumonectomy surgery is corroborated in Asian patients in Samsung Medical Center where patients who achieved pathologic downstaging of N2 disease following neoadjuvant CRT achieved improved outcomes irrespective of initial cN2 bulk/extent [27,28].

The Swiss phase III randomized (SAKK) trial enrolled 232 patients with stage IIIA/N2 NSCLC into two treatment groups: in CHRT group three cycles of neoadjuvant cisplatin and docetaxel, was followed by RT with 44 Gy in 22 fractions over 3 weeks. The control group received the same CHT alone, and all patients were scheduled to undergo surgery. Overall tumor RR was $61 \%$ vs. 44\% ( $\mathrm{P}=0.012)$ in CHRT and CHT alone groups respectively with acceptable toxicity [29]. Nodal downstaging (to N1 or N0) was observed in $64 \%$ and 53\%, respectively, and the pCR in $16 \%$ and $12 \%$, respectively in the CHRT group and CHT only group $(\mathrm{P}=\mathrm{NS})$. The median event-free survival was 12.8 months vs 11.6 months and MST were 37.1 months and 26.2 months in the CHRT and control group, respectively, but survivals at 2-4 years were identical in the 2 treatment arms. Thus, this study showed that patients who received CHRT before surgery had an objective response, a pCR, a R0 resection rate and a mediastinal downstaging more frequently and less local progression than patients in the CHT alone group. In spite of all of these, the addition of RT neither improved event-free survival nor OS. Authors concluded that one definitive local treatment modality combined with neoadjuvant CHT might have been sufficient [29].

To improve results some trials focused on head-to-head comparison of various treatment sequences. A Japanese phase III randomized trial for stage IIIA/N2 NSCLC compared induction concurrent CHRT with carboplatin and docetaxel followed by surgery to induction CHT followed by surgery [30]. The study had to be stopped because of slow accrual, after 60 patients were randomized. Combined CHRT conferred a better local control, however there was no differences in PFS or OS when RT was added to CHT. Treatments were well tolerated without any toxic deaths [30].

Two recent meta-analyses attempted to answer the question of superior neoadjuvant treatment, i.e. neoadjuvant CHT followed by surgery versus neoadjuvant CHRT followed by surgery [31,32]. Shah et al. [31] identified seven studies, of which there was only one fully published RCT, and one Phase II randomized trial. Neither of the studies showed a survival benefit for induction CHRT and results remained the same when analysis was confined to two RCTs (one Phase III and one Phase II). It must, however, clearly be said that the inclusion of retrospective papers, and data presented in abstract form only, clearly limited the quality of this meta-analysis. In their meta-analysis, Xu Y-P et al. [32] found that induction CHRT before surgical resection significantly increased the rate of pCR in resected mediastinal lymph nodes compared with those who received induction CHT, but there was no significant difference in tumour downstaging, OS or PFS. A recent prospective RCT [29] from SAKK which was not included in either meta-analysis confirmed these findings.

Unfortunately, still we do not have any predictive or prognostic factors for selecting surgery in the treatment of patients with stage IIIA/pN2 NSCLC. Jeremic et al did not identify any study investigating treatment-related predictive factors for the potential superiority of surgical outcomes in patients with Stage IIIA (mostly pN2) NSCLC. These authors concluded that currently it was impossible to identify any treatment-related predictive or prognostic factors for selecting surgery in patients with stage IIIA/pN2 NSCLC [33].

Patients with residual nodal disease (RND)-particularly positive mediastinal adenopathy-typically fare much worse. Important question whether pathologic response should guide the decision to proceed with surgical resection. Ziel et al. investigated nodal pathologic complete response (N-PCR) as a strong predictor of OS. They compared the outcomes of patients treated with trimodality treatment (TMT) versus CHRT, focusing on the importance of N-PCR. Actuarial OS, PFS, and distant metastasis-free survival (DMFS) were compared between patients treated with CHRT and TMT and between CHRT and N-PCR/RND. On multivariable analyses, N-PCR had superior OS (hazard ratio [HR], 0.38; $\mathrm{p}=0.0012$ ), PFS (HR, 0.42; $\mathrm{p}=0.0005)$, and DMFS (HR, $0.42 ; p=0.0007)$ compared with CHRT. Conversely, there were trends for worse OS and PFS for RND versus CHRT, although only inferior DMFS was significant (HR, 1.83; $p=0.04)$. Study showed that surgical patients with N-PCR experienced superior survival, but those with RND fared no better than CHRT alone. Authors concluded that mediastinal response might play an important role in the decision to proceed with surgical resection after CHRT for stage III NSCLC [34].

In conclusion, neoadjuvant CHRT is safe and efficient, with higher overall clinical response, pCR rates and a higher mediastinal clearing compared to neoadjuvant CHT alone. On the other hand, the yet available randomized studies have failed to demonstrate any advantage of adding RT in the neoadjuvant setting regarding PFS or OS. Admittedly the number of patients enrolled was modest.

\section{Neoadjuvant CHT followed by CRT vs Surgery}

Van Meerbeeck et al performed a large multicenter prospective randomized trial in patients with stage IIIA-N2 NSCLC who showed a response to three cycles of platinum-based induction CHT. Responding patients were subsequently randomly assigned to surgical resection or RT. This large randomized multicenter study demonstrated that surgery did not improve survival after a radiologic response to induction $\mathrm{CHT}$ in patients with unresectable 
stage IIIA-N2 NSCLC as compared with RT [35]. These results were important because several centers continued to routinely use induction CHT followed by surgery to treat patients with this stage of disease based on small, randomized studies that showed that surgery alone is inferior to perioperative chemotherapy and surgery in stage IIIA patients [36-38].

ESPATUE study randomized patients after 3 cycles of induction cisplatin- paclitaxel and concurrent CHRT with a cisplatin and vinorelbine with 45 Gy in 1.5Gy fractions BID followed by definitive RT boost dose of 20 - 26Gy in 2Gy fractions or surgical resection [14]. There was no difference in 5-year OS (40\% vs. $44 \%, \mathrm{p}=0.34$ ) or PFS ( $35 \%$ or $32, \mathrm{p}=0.75$ ) between the CHRT and surgical arms, respectively [39]. Results of these studies were the subject of four meta-analyses/systematic reviews [31,40-42] which were summarized by Jeremic et al. [43] who clearly showed that there is no place for surgery in a multimodality treatment in these patients. The most recent meta-analysis of Pottgen et al. also analyzed randomized trials comparing surgery with definitive RT as local curative treatment options within the framework of different multimodality treatments for patients with locally advanced (LA-NSCLC). Endpoints for comparison of treatment results were OS, PFS, and toxicity. This meta-analysis also did not find significantly different OS in patients with LA-NSCLC after induction treatment and surgery compared with those receiving definitive CHRT. Trials using concurrent CHRT in the neoadjuvant phase of the resection arm and simultaneous CHRT in the other arm showed a better OS than the other trials using induction CHT alone before surgery in the surgical arm and sequential CHT and RT or RT alone in the other [44].

Additional evidence came from the recent study which analyzed NCDB data from 1,076 patients with stage IIIA (N2) NSCLC, treated between 2003 to 2005, either with neo-adjuvant CHRT followed by surgery or neoadjuvant CHT followed by surgery. The 5-year OS for the entire cohort was 39\%, namely $39.2 \%$ for the neo-adjuvant CHRT category vs. $38.6 \%$ for the neoadjuvant CHT (P= NS). On multivariate analysis, neo-adjuvant CHRT was associated with an improved pathological outcome [45].

\section{Neoadjuvant immunotherapy Followed by Surgery}

Currently, a number of trials are studying neoadjuvant immunotherapy, mainly in stages IB-IIIA. One of the first studies (NADIM trial) testing combination CHT and immunotherapy as induction therapy in stage IIIA patients with histologic N2 confirmation enrolled 46 patients who received 3 cycles of paclitaxel/carboplatin plus nivolumab before surgical resection, followed by adjuvant nivolumab monotherapy for 1 year. Major pathologic response (MPR) was achieved in $82.9 \%$ of patients, $63.4 \%$ of whom had pCR. Down-staging was achieved in $90.2 \%$ and $O S$ at 20 months was $90 \%$ [46]. In a randomized phase II study (NEOSTAR trial) of induction checkpoint blockade for patients with untreated stage I-IIIA (single N2) NSCLC, patients received three doses of nivolumab $(3 \mathrm{mg} / \mathrm{kg}$ ) or nivolumab ( $3 \mathrm{mg} / \mathrm{kg}$ ) plus ipilimumab ( $1 \mathrm{mg} / \mathrm{kg}$ ) every 2 weeks followed by surgery. 39 of 44 patients underwent surgery (89\% resectability). Major pathologic response (MPR) rate was 24\% overall; $17 \%$ with nivolumab and $33 \%$ with the combination therapy [47].

The results of several studies of CHT and immunotherapy with surgery are awaited. NCT 02716038 study of neoadjuvant four cycles of atezolizumab plus carboplatin and nabpaclitaxel reported an MPR of 64\% in 11 patients who underwent resection [48]. KEYNOTE-671 trial (NCT 03425643) investigates efficacy and safety of Pembrolizumab with platinum doublet as neoadjuvant/adjuvant therapy for patients with resectable stage IIB/IIIA NSCLC [49]. CheckMate-816 (NCT 02998528), a phase III open-label study, compares EFS and pCR rates among participants treated with neoadjuvant nivolumab plus platinum doublet $\mathrm{CHT}$ versus platinum doublet $\mathrm{CHT}$ alone in stage IB-IIIA NSCLC [50]. IMpower-030 (NCT03456063) trial with the surrogate objective MPR as its primary endpoint, randomized patients between four cycles of platinum doublet CHT with atezolizumab or placebo, followed by surgery and postoperative standard of care in stage II-IIIAIIIB (T3N2) disease [51]. 77T trial (NCT04025879) is comparing neoadjuvant chemoimmunotherapy with nivolumab to neoadjuvant CHT plus placebo, followed by surgical removal and adjuvant treatment with nivolumab or placebo including patients with suspected or histologically confirmed, resectable stage IIA-IIIB NSCLC. The primary endpoint is EFS as assessed by blinded independent central review [52]. A randomized, two-arm, phase II, multi-center study (NADIMII trial) compares neoadjuvant chemoimmunotherapy to neoadjuvant CHT alone. Patients in the experimental arm, receive nivolumab plus paclitaxel plus carboplatin for 3 cycles as neoadjuvant treatment followed by surgery and 6 months of adjuvant treatment with nivolumab. The primary objective is pCR [53]. Many of these studies include very different populations, which makes it difficult to draw firm conclusions for diverse stages or presentations. Homogeneous populations should be studied in very limited scenarios designed to answer a specific question. In addition, no molecular markers predicting increased survival and/or response in the immunotherapy setting have been reported to date. Longer follow up evaluating PFS and OS are urgently needed.

\section{Conclusion}

Still the controversy is not being solved and further trials considering a better patients' selection, innovative RT and more efficient systemic treatments need to be undertaken. Selection of patients for a surgical resection is challenging, due to both patient (comorbidity, refusal) and tumor (no downsizing or downstaging) related factors. It seems that studies with chemoimmunotherapy provide a higher rate of pathologic responses and downstaging 


\section{Cancer Therapy \& Oncology International Journal}

than previous studies conducted solely with CHT. On the other hand, it remains to be confirmed whether pCR is a sufficient surrogate for OS. Hopefully, the next two decades will bring much needed improvements for our patients.

\section{Acknowledgement}

\section{$\mathrm{N} / \mathrm{A}$}

\section{Conflict of Interest}

The Authors Declare no Competing Interests Regarding this Study.

\section{References}

1. Ettinger DS, Wood DE, Aggarwal C, Aisner DL, Akerley W, et al. (2019) NCCN Guidelines Insights: Non-Small Cell Lung Cancer, Version 1.2020. J Natl Compr Canc Netw 17(12): 1464-1472.

2. Mirimanoff RO (2015) Neoadjuvant chemoradiotherapy followed by surgery for stage IIIa and IIIb non-small-cell lung cancer (NSCLC): is it still justified? Chin Clin Oncol 4(4): 49.

3. Rosell R, Gomez-Codina J, Camps C, Maestre J, Padille J, Canto A, et al. (1994) A randomized trial comparing preoperative chemotherapy plus surgery with surgery alone in patients with non-small-cell lung cancer. N Engl J Med 330(3): 1153 -1158.

4. Roth JA , Fossella F , Komaki R, Ryan MB , Putnam JB Jr , et al. (1994) A randomized trial comparing perioperative chemotherapy and surgery with surgery alone in resectable stage IIIA non-small-cell lung cancer. J Natl Cancer Inst 86(9): 673-680.

5. Pass HI, Pogrebniak HW, Steinberg SM, Mulshine J, Minna J (1992) Randomized trial of neoadjuvant therapy for lung cancer: interim analysis . Ann Thorac Surg 53(6): 992 -998.

6. Gilligan D, Nicolson M, Smith I, Groen H, Dalesio O, et al. (2007) Preoperative chemotherapy in patients with resectable non-small cell lung cancer: results of the MRC LU22/NVALT 2/EORTC 08012 multicentre randomised trial and update of systematic review. Lancet 369(9577): 1929-1937.

7. NSCLC Meta-analysis Collaborative Group (2014) Preoperative chemotherapy for non-small-cell lung cancer: a systematic review and meta-analysis of individual participant data. Lancet 383(9928): 15611571.

8. Song WA, Zhou NK, Wang W, Xiang-Yang Chu, Chao-Yang Liang, et al. (2015) Survival benefit of neoadjuvant chemotherapy in non-small cell lung cancer: an updated meta-analysis of 13 randomized control trials. J Thorac Oncol 5(4): 510-516.

9. Berghmans T, Paesmans M, Meert AP, C Mascaux, P Lothaire, et al. (2005) Survival improvement in resectable non-small cell lung cancer with (neo)adjuvant chemotherapy: results of a meta-analysis of the literature. Lung Cancer 49(1): 13-23.

10. Nakamura H, Kawasaki N, Taguchi M, Kabasawa K (2006) Role of preoperative chemotherapy for non-small-cell lung cancer: a metaanalysis. Lung Cancer 54(3): 325-329.

11. Burdett S, Stewart LA, Rydzewska L (2006) A systematic review and meta-analysis of the literature: chemotherapy and surgery versus surgery alone in non-small cell lung cancer. J Thorac Oncol 1(7): 611621.

12. Lim E, Harris G, Patel A, Adachi I, Edmonds L, et al. (2009) Preoperative versus postoperative chemotherapy in patients with resectable nonsmall cell lung cancer: systematic review and indirect comparison meta-analysis of randomized trials. J Thorac Oncol 4(11): 1380-1388.

13. Felip E, Rosell R, Maestre JA, José Manuel Rodríguez-Paniagua, Teresa Morán, et al. (2010) Preoperative chemotherapy plus surgery versus surgery plus adjuvant chemotherapy versus surgery alone in earlystage non small-cell lung cancer. J Clin Oncol 28(19): 3138-3145.

14. Pisters KM, Vallières E, Crowley JJ, Wilbur A Franklin, Paul A Bunn Jr, et al. (2010) Surgery with or without preoperative paclitaxel and carboplatin in early stage non-small-cell lung cancer: Southwest Oncology Group Trial S9900, an intergroup, randomized, phase III trial. J Clin Oncol 28(11): 1843-1849.

15. Machtay M, Lee JH, Stevenson JP, Joseph B Shrager, Kenneth M Algazy, et al. (2004) Two commonly used neoadjuvant chemoradiotherapy regimens for locally advanced stage III non-small cell lung carcinoma: longtermresults and associations with pathologic response. J Thorac Cardiovasc Surg 127: 108-113.

16. Stamatis G, Eberhardt W, Pöttgen C (2004) Surgery after multimodality treatment for non-small-cell lung cancer. Lung Cancer 45 Suppl 2: S107-S112.

17. Pezzetta E, Stupp R, Zouhair A, Louis Guillou, Patrick Taffé, et al. (2005) Comparison of neoadjuvant cisplatin-based chemotherapy versusradiochemotherapy followed by resection for stage III (N2) NSCLC. Eur J Cardiothorac Surg 27(6): 1092-1098.

18. Higgins K, Chino JP, Marks LB, Neal Ready, Thomas A D'Amico, et al. (2009) Preoperativechemotherapy versus preoperative chemoradiotherapyfor stage III (N2) non-small-cell lung cancer. Int J RadiatOncol Biol Phys 75: 1462-1467.

19. Kim AW, Liptay MJ, Bonomi P, William H Warren, Sanjib Basu, et al. (2011) Neoadjuvantchemoradiation for clinically advanced non-smallcellung cacner: an analysis of 233 patients. Ann Thorac Surg 92(1): 233-241; discussion 241-243.

20. Paul S, Mirza F, Port JL, Paul C Lee, Brendon M Stiles, et al. (2011) Survival of patients with clinical stage IIIA non-small-cell lung cancer afterinduction therapy: age, mediastinal downstaging, and extent of pulmonary resection as independant predictors. J Thorac Cardiovasc Surg 141(1): 48-58.

21. Shumway D, Corbin K, Salgia R, R M Malik, D J Haraf, et al. (2011) Pathologicresponse rates following definitve dose image-guided chemoradiotherapy and resection for locally advancednon-smallcelllung cancer. Lung Cancer 74(3): 446-450.

22. Pöttgen C, Eberhardt W, Graupner B, D Theegarten, T Gauler, et al. (2013) Accelerated hyperfractionated radiotherapy within trimodality therapyconcepts for stage IIIA/B non-small-cell lung cancer: Markedly higher rates of pathologic complete remissionsthan with conventional fractionation. Eur J Cancer 49(9): 2107-2115.

23. Thomas M, Rübe C, Hoffknecht P, Macha HN, Freitag L, et al. (2008) Effect of preoperative chemoradiation in addition to preoperative chemotherapy: a randomised trial in stage III non-small-cell lung cancer. Lancet Oncol 9(7): 636-648.

24. Albain KS, Swann RS, Rusch VW, Turrisi AT 3rd, Shepherd FA, et al. (2009) Radiotherapy plus chemotherapy with or without surgical resection for stage III non-small-cell lung cancer: a phase III randomised controlled trial. Lancet 374(9687): 379-386.

25. Yamaguchi M, Toyokawa G, Ohba T, Tomonari Sasaki, Takuro Kometani, et al. (2013) Preoperative concurrent chemoradiotherapy of S-1/cisplatin for stage III non-small cell lung cancer. Ann Thorac Surg 96(5): 1783-1789.

26. Kato M, Onishi H, Matsumoto K, Nobuko Tsuruta, Kazuyuki Higuchi, et al. (2012) Preoperative chemoradiotherapy using cisplatin plus S-1 can induce downstaging in patients with locally advanced (stage III) non small-cell lung cancer. Anticancer Res 32(11): 5099-5104. 


\section{Cancer Therapy \& Oncology International Journal}

27. Kim HK, Cho JH, Choi YS, Jae Ill Zo, Young Mog Shim, et al. (2016) Outcomes of neoadjuvant concurrent chemoradiotherapy followed by surgery for non-small-cell lung cancer with N2 disease. Lung Cancer 96: 56-62.

28. Lee H, Ahn YC, Pyo H, Bokyong Kim, Dongryul Oh, et al. (2014) Pretreatment clinical mediastinal nodal bulk and extent do not influence survival in N2-positive stage IIIA non-small cell lung cancer patients treated with trimodality therapy. Ann Surg Oncol 21(6): 2083-2090.

29. Pless M, Stupp R, Ris HB, Stahel RA, Weder W, et al. (2015) Induction chemoradiation in stage IIIA/N2 non-small-cell lung cancer: a phase 3 randomised trial. Lancet 386(9998): 1049-1056.

30. Katakami N, Tada H, Mitsudomi T, Shinzoh Kudoh, Hiroshi Senba, et al. (2012) A phase 3 study of induction treatment with concurrent chemoradiotherapyversus chemotherapy before surgery in patients with pathologically confirmed N2 stage IIIA non-small cell lung cancer (WJTOG9903). Cancer 118(24): 6126-6135.

31. Shah AA, Berry MF, Tzao C, Mihir Gandhi, Mathias Worni, et al. (2012) Induction chemoradiation is not superior to induction chemotherapy alone in stage IIIA lung cancer. Ann Thorac Surg 93: 1807-1812.

32. Xu Y-P, Li B, Xu X-L, Wei-Min Mao, et al. (2015) Is there a survival benefit in patients with Stage IIIA (N2) non-small cell lung cancer receiving neoadjuvant chemotherapy and/or radiotherapy prior to surgical resection. A systematic review and meta-analysis. Medicine (Baltimore) 94(23): e879.

33. Jeremić B, Casas F, Dubinsky P, Gomez-Caamano A, Čihorić N, et al. (2016) Surgery for Stage IIIA Non-Small-cell Lung Cancer: Lack of Predictive and Prognostic Factors Identifying Any Subgroup of Patients Benefiting From It. Clin Lung Cancer 17(2): 107-112.

34. Ziel E, Hermann G, Sen N, Bonomi P, Liptay MJ, et al. (2015) Survival Benefit of Surgery after Chemoradiotherapy for Stage III (N0-2) NonSmall-Cell Lung Cancer Is Dependent on Pathologic Nodal Response. J Thorac Oncol 10(10): 1475-1480.

35. van Meerbeeck JP, Kramer GW, Van Schil PE, Catherine Legrand, Egbert F Smit, et al. (2007) Randomized controlled trial of resection versus radiotherapy after induction chemotherapy in stage IIIA-N2 nonsmall-cell lung cancer. J Natl Cancer Inst 99(6): 442-450.

36. Rosell R, Gomez-Codina J, Camps C, Maestre J, Padille J, Canto A, et al. (1994) A randomized trial comparing preoperative chemotherapy plus surgery with surgery alone in patients with non-small-cell lung cancer . N Engl J Med 330: 1153-1158.

37. Roth JA, Fossella F, Komaki R, Ryan MB, Putnam JB Jr, et al. (1994) A randomized trial comparing perioperative chemotherapy and surgery with surgery alone in resectable stage IIIA non-small-cell lung cancer. J Natl Cancer Inst 86(9): 673-680.

38. Pass HI, Pogrebniak HW, Steinberg SM, Mulshine J, Minna J (1992) Randomized trial of neoadjuvant therapy for lung cancer: interim analysis . Ann Thorac Surg 53(6): 992-998.

39. Eberhardt WE, Pöttgen C, Gauler TC, Friedel G, Veit S, et al. (2015) Phase III Study of Surgery Versus Definitive Concurrent Chemoradiotherapy Boost in Patients with Resectable Stage IIIA(N2) and Selected IIIB NonSmall-Cell Lung Cancer After Induction Chemotherapy and Concurrent Chemoradiotherapy (ESPATUE). J Clin Oncol 33(35): 4194-201.

40. Ren Z, Zhou S, Liu Z, Shaofa Xu, et al. (2015) Randomized controlled trials of induction treatment and surgery versus combined chemotherapy and radiotherapy in stages IIIA-N2 NSCLC: a systematic review and meta-analysis. J Thorac Dis 7(8): 1414-1422.
41. McElnay PJ, Choong A, Jordan E, F Song, E Lim, et al. (2015) Outcome of surgery versus radiotherapy after induction treatment in patients with N2 disease: systematic review and meta-analysis of randomised trials. Thorax 70(8): 764-768

42. Xu X-L, Dan L, Chen W, Shuang-Mei Zhu, Wei-Min Mao, et al. (2016) Neoadjuvant chemoradiotherapy or chemotherapy followed by surgery is superior to that followed by definitive chemoradiation or radiotherapy in stage IIIA(N2) nonsmall-cell lung cancer: a metaanalysis and system review. OncoTargets Ther 9: 845-853.

43. Jeremic B, Casas F, Dubinsky P, Gomez-Caamano A, Cihoric N, et al (2017) Combined modality therapy in Stage IIIA nonsmall cell lung cancer: clarity or confusion despite the highest level of evidence? J Radiat Res 58(3): 267-272.

44. Pöttgen C, Eberhardt W, Stamatis G, Stuschke M (2017) Definitive radiochemotherapy versus surgery within multimodality treatment in stage III non-small cell lung cancer (NSCLC) - a cumulative metaanalysis of the randomized evidence. Oncotarget 8(25): 41670-41678.

45. Sher DJ, Fidler MJ, Liptay MJ, Matthew Koshy (2015) Comparative effectiveness of neoadjuvant chemoradiotherapy versus chemotherapy alone followed by surgery for patients with stage IIIA non-small cell lung cancer. Lung Cancer 88(3): 267-274.

46. Provencio M, Nadal E, Insa A, E Nadal, A Insa, et al. (2019) NADIM Study: Updated Clinical Research and Outcomes. Presented at IASLC World Conference on Lung Cancer; Barcelona, Spain 14(10).

47. Cascone T, Williams WN, Weissferdt A, Heather Y Lin, Cheuk Hong Leung, et al. (2019) Neoadjuvant nivolumab (N) or nivolumab plus ipilimumab (NI) for resectable non-small cell lung cancer (NSCLC): Clinical and correlative results from the NEOSTAR study. J Clin Oncol 37(no. 15_suppl): 8504.

48. Shu CA, Gainor JF, Awad MM, Chiuzan C, Grigg CM, et al. (2020) Neoadjuvant atezolizumab and chemotherapy in patients with resectable non-small-cell lung cancer: an open-label, multicentre, single-arm, phase 2 trial. Lancet Oncol 21(6): 786-795.

49. Efficacy and Safety of Pembrolizumab (MK-3475) With Platinum Doublet Chemotherapy as Neoadjuvant/Adjuvant Therapy for Participants With Resectable Stage II, IIIA, and Resectable IIIB (T34N2) Non-small Cell Lung Cancer (MK-3475-671/KEYNOTE-671).

50. A Neoadjuvant Study of Nivolumab Plus Ipilimumab or Nivolumab Plus Chemotherapy Versus Chemotherapy Alone in Early Stage Non-Small Cell Lung Cancer (NSCLC) (CheckMate 816).

51. A Study of Neoadjuvant Atezolizumab Plus Chemotherapy Versus Placebo Plus Chemotherapy in Patients With Resectable Stage II, IIIA, or Select IIIB Non-Small Cell Lung Cancer (IMpower030).

52. A Phase 3, Randomized, Double-blind Study of Neoadjuvant Chemotherapy Plus Nivolumab Versus Neoadjuvant Chemotherapy Plus Placebo, Followed by Surgical Resection and Adjuvant Treatment with Nivolumab or Placebo for Participants With Resectable Stage IIIIIB Non-small Cell Lung Cancer.

53. A Randomized Phase II Study of Neo-adjuvant Chemo/Immunotherapy Versus Chemotherapy Alone for the Treatment of Locally Advanced and Potentially Resectable Non-small Cell Lung Cancer (NSCLC) Patients. 
Your next submission with Juniper Publishers will reach you the below assets

- Quality Editorial service

- Swift Peer Review

- Reprints availability

- E-prints Service

- Manuscript Podcast for convenient understanding

- Global attainment for your research

- Manuscript accessibility in different formats ( Pdf, E-pub, Full Text, Audio)

- Unceasing customer service

Track the below URL for one-step submission https://juniperpublishers.com/online-submission.php 